\title{
OPEN Venom duct origins of prey capture and defensive conotoxins in piscivorous Conus striatus
}

\author{
S. W. A. Himaya ${ }^{1}$, Ai-Hua Jin ${ }^{1,4}$, Brett Hamilton ${ }^{2,4}$, Subash K. Rai ${ }^{1,3,4}$, Paul Alewood ${ }^{1}$ \& \\ Richard J. Lewis ${ }^{1 凶}$
}

The venom duct origins of predatory and defensive venoms has not been studied for hook-and-line fish hunting cone snails despite the pharmacological importance of their venoms. To better understand the biochemistry and evolution of injected predatory and defensive venoms, we compared distal, central and proximal venom duct sections across three specimens of $C$. striatus (Pionoconus) using proteomic and transcriptomic approaches. A total of 370 conotoxin precursors were identified from the whole venom duct transcriptome. Milked defensive venom was enriched with a potent cocktail of proximally expressed inhibitory $\alpha$-, $\omega$ - and $\mu$-conotoxins compared to milked predatory venom. In contrast, excitatory $\mathrm{KA}$-conotoxins dominated both the predatory and defensive venoms despite their distal expression, suggesting this class of conotoxin can be selectively expressed from the same duct segment in response to either a predatory or defensive stimuli. Given the high abundance of KA-conotoxins in the Pionoconus clade, we hypothesise that the KA-conotoxins have evolved through adaptive evolution following their repurposing from ancestral inhibitory $A$ superfamily conotoxins to facilitate the dietary shift to fish hunting and species radiation in this clade.

Cone snails are predatory marine gastropods that have evolved one of the most sophisticated envenomation strategies known, supporting their explosive radiation into over 850 species $^{1}$. Conus venoms typically contain thousands of mostly disulfide-rich and highly structured peptides called conotoxins that target a wide range of neuromuscular receptors, ion channels and transporters ${ }^{2,3}$ to facilitate prey capture and for defence against predators $^{4-7}$. Cone snails typically prey on worms (vermivorous), other molluscs (molluscivorous) or fish (piscivorous), with the predatory venoms of fish hunters evolved to target vertebrate receptors and ion channels.

Defensive venoms in worm hunting species appear to have facilitated the dietary shift to fish hunting in cone snails $^{4,8}$. Currently 8 subgenera (clades) of cone snails are classified as fish hunters (Pionoconus, Chelyconus, Gastridium, Textilla, Phasmoconus, Embrikena, Alfonsoconus and Asprella) of the 68 identified clades, although direct fish hunting observations are missing in the Embrikena, Alfonsoconus and Asprella clades ${ }^{9-11}$. Fish hunting is proposed to have evolved from ancestral worm hunting cone snail species ${ }^{4,12,13}$ through the repurposing of defensive venom peptides ${ }^{4}$. Among these fish hunters, the Pionoconus clade is widely distributed, accounting 40 known fish hunting species ${ }^{11}$. Venoms of only a few species of Pionoconus clade (C. magus, C. catus, C. consors and $C$. striatus) have been studied extensively due to their medical potential ${ }^{14-17}$. These include the first marine drug $\omega$-conotoxin MVIIA found in C. magus ${ }^{18}$, the related $\omega$-conotoxin CVID from C. catus that showed efficacy in clinical trials ${ }^{19}$, and the potentially more selective CVIE and CVIF that have been investigated pre-clinically ${ }^{20}$.

The venom of cone snails is highly variable both between and within species ${ }^{16,21-25}$ as well as spatially along the venom duct ${ }^{4,9,26}$. Spatial differentiation in the venom gland has been shown to correlate with functionally distinct predatory and defensive venoms for the net feeding fish hunter C. geographus ${ }^{4}$. However, differentiation of defensive and predatory venoms in hook-and-line fish hunting clades and their venom duct origins has not been investigated. In this study, we used a combination of proteomics and transcriptomic approaches to decode the venom profiles of C. striatus from the Pionoconus clade. Characterisation of conotoxins between three individuals and their trisected venom ducts revealed differential expression amongst the 25 known superfamilies identified. The $\kappa \mathrm{A}$-conotoxins of the A superfamily and conkunitzins dominated the distal venom duct, while $\alpha$-like conotoxins of the A superfamily, $\omega$ - and $\delta$-like conotoxins of the $\mathrm{O} 1$ superfamily, $\mu$-like conotoxins of the $\mathrm{M}$ superfamily, contryphans and con-ikot-ikots dominated the proximal venom duct. Analysis of the milked

\footnotetext{
${ }^{1}$ Institute for Molecular Bioscience, The University of Queensland, Saint Lucia, OLD 4072, Australia. ${ }^{2}$ Centre for Microscopy and Microanalysis, The University of Queensland, Saint Lucia, QLD 4072, Australia. ${ }^{3}$ Present address: Genome Innovation Hub, The University of Queensland, Saint Lucia, OLD 4072, Australia. ${ }^{4}$ These authors contributed equally: Ai-Hua Jin, Brett Hamilton and Subash K. Rai. ${ }^{\circledR}$ email: r.lewis@uq.edu.au
} 
predatory and defensive venoms revealed that the distally dominant $\kappa \mathrm{A}$-conotoxins were expressed in response to both predatory and defensive stimuli. Findings of this study, together with previously published reports, suggest $\mathrm{kA}$-conotoxins might be the key evolutionary drivers of successful radiation in Pionoconus clade cone snails.

\section{Results and discussion}

Transcriptomics reveals $C$. striatus venom complexity. Nine transcriptomes from trisected venom ducts ( $\sim 4 \mathrm{~cm}$ distal, central and proximal sections) of three adult specimens (A, B and C) of C. striatus were obtained using NextSeq 300 cycle $(2 \times 150 \mathrm{bp})$ high output Illumina run (Table 1$)$. Although this platform facilitates deep sequence analysis with a significantly low error rate $(\sim 0.1 \%)$, the relatively short read lengths $(150 \mathrm{bp}$ paired end) makes de novo transcript assembly an essential step to obtain full conotoxin transcript sequences ( $>400$ bp). A rationally designed in-house optimised de-novo assembly method using Trinity platform with conotoxin precursor (complete conotoxin transcript) annotation was used to unravel the C. striatus venom repertoire (supplementary Table S1). In comparison to default Trinity assembly parameters, the optimised method applied two kmers of sizes of 19 and 31 to capture both low and highly expressed transcripts, with SNP transcripts maintained and redundant transcripts removed during assembly. These parameters improved the capture of transcriptomic messiness ${ }^{27}$ and identified superfamilies and unique transcripts that were missed using the default parameters of the Trinity platform (supplementary Table S2).

Comparison of venom duct transcriptomes. A total of 370 unique conotoxin precursors were classified into 25 superfamilies based on the signal sequence similarity $(>60 \%)$ across the three specimens of $C$. striatus (supplementary Table S3). Despite the variety of gene superfamilies found, no new conotoxin precursor signal sequences were detected. However, high levels of variation in mature conotoxin sequences were observed, with only $11 \%$ (42) of the transcripts present in all three transcriptomes, only $29.5 \%$ (109) of transcripts shared between at least two specimens, leaving 261 unique transcripts identified form the three specimens (Fig. 1A). Only 21 of the 72 previously identified conotoxins from C. striatus were found in these three specimens (supplementary Table S3 and S4). This high level of intraspecific conotoxin diversity in C. striatus is reminiscent of the levels of variation reported in other species of cone snails from fish hunting Pionoconus and Chelyconus clades ${ }^{16,24,25,28}$.

Despite the variability in conotoxin precursors, the superfamily profiles of the three specimens were similar (Fig. 1B and supplementary Table S5). All three C. striatus transcriptomes were dominated by transcripts from superfamilies A, O1, O2, M, conkunitizin, contryphan and con-ikot-ikot, with the A and O1 superfamilies accounting for $>80 \%$ of the total conotoxin transcript expression in each specimen. In contrast, the number of transcripts from minor superfamilies was more varied (supplementary Table S5), while the O2 and M superfamilies had high sequence diversity despite their relatively low expression levels (Fig. 1C). This structural and functional conservation within superfamilies observed in C. striatus has been seen in other fish hunting species $^{16,17,26}$, suggesting that diversifying selection around structurally and functionally conserved motifs underpins the evolution of conotoxin diversity in fish hunting cone snails.

Superfamily compartmentalisation. Analysis of the conotoxin precursor transcript profiles across trisected distal, central and proximal venom duct sections revealed a clear compartmentalisation for the dominant superfamilies A, O1, O2, contryphan, conkunitzin, M and con-ikot-ikot (Fig. 2A, supplementary Table S6). Similar compartmentalisation was observed across all three specimens, suggesting that the conotoxin distribution pattern along the venom duct has functional significance. In contrast, the low and inconsistent expression levels obscured identification of any clear compartmentalisation patterns for the remaining 16 minor superfamilies. A superfamily $\kappa$ A-conotoxins of framework IV (A-IV) showed preferential expression in the distal venom duct (Fig. 2B), accounting for $77.3 \%, 43.2 \%$ and $56.2 \%$ of total relative expression distally (Fig. 2A, supplementary Figure $\mathrm{S} 1$ ) that decreased to $0.5 \%, 0.14 \%$ and $1.83 \%$ in the proximal section of specimens $\mathrm{A}, \mathrm{B}$ and $\mathrm{C}$, respectively. KA-SIVA and SIVB were the most abundant peptide precursors in all three distal transcriptomes. Similarly, conkunitzin precursors were common in the distal transcriptomes $(\sim 6 \%)$, while they represented $<0.1 \%$ of expression in the proximal duct sections of all three specimens. In contrast, expression levels of putative A-I and II $\alpha$-conotoxins, O1, M-III and con-ikot-ikot superfamilies increased proximally in the venom duct transcriptomes of all specimens (Fig. 2A, supplementary Figure S1). Interestingly, A-I and A-II sub-families comprised $\sim 50 \%$ of toxin expression in the proximal sections of specimens A and C ( $53 \%$ and $54.43 \%$, respectively), but were relatively common in the distal portions in all specimens (7\%,20\% and $8 \%$, respectively). The increase in A-I and A-II toxin expression towards the proximal region is gradual, with the central section transcriptome being transitionary (Fig. 2A, supplementary Figure S1). Among A-I conotoxins, $\alpha$-SI with 3/5 cysteine architecture targeting muscular nicotinic receptors was the most abundant A-I precursor (in all specimens), while $\alpha$-SII precursor is the only A-II conotoxin present in all three specimens. Interestingly, a-SII was the most abundant conotoxin in the proximal transcriptomes of specimens A and B while a-SI dominates the proximal transcriptome of specimen C.

The O1 superfamily transcripts were dominant in both central $(40.7 \%, 44.7 \%, 64.1 \%)$ and proximal $(33.3 \%$, $54.2 \%, 30.1 \%)$ transcriptomes compared to the distal transcriptomes $(8.5 \%, 24.3 \% 22.3 \%)$ in all three specimens. Despite these differences in the number of precursors identified, the O1 superfamily had similarly high sequence numbers and diversity across all the sections of the venom duct (supplementary Table S6). Based on the number of sequences identified, O1 was the most dominant superfamily across the venom duct, with 64 novel and 16 known $\omega$ - and $\delta$-like conotoxins (supplementary Table S3). O1 superfamily precursor number increased proximally, except $\omega$-conotoxin SVIB and the $\delta$-like conotoxin S6.8 that were highly expressed in the distal transcriptomes but insignificant in the proximal transcriptomes of the three specimens (Fig. 3A). Contryphans were the 


\begin{tabular}{|c|c|c|c|c|c|}
\hline Sequence ID & Superfamily & Mature sequence $^{*}$ & Distal & Central & Proximal \\
\hline STR1_SI & A & ICCNPACGPKYSC & $\checkmark$ & $\checkmark$ & $\checkmark$ \\
\hline STR14_Sm1.2 & A & NGCCRNPACESHRC & & $\checkmark$ & $\checkmark$ \\
\hline STR17_SII & A & GCCCNPACGPNYGCGTSCS & $\checkmark$ & $\checkmark$ & $\checkmark$ \\
\hline STR18 & A & APALVVTATTNCCGYTGPACHPCLCTQTC & $\checkmark$ & & \\
\hline STR19 & A & QKELVPSVITTCCGYDPGTMCPPCRCDNSCKPKPKK & $\checkmark$ & & \\
\hline STR20_SIVB & A & QKELVPSVITTCCGYDPGTMCPPCRCTNSC & $\checkmark$ & & \\
\hline STR21_SIVA & A & QKSLVPSVITTCCGYDPGTMCPPCRCTNSC & $\checkmark$ & $\checkmark$ & \\
\hline STR22 & A & QKSLVPSVITTCCGYDPGTMCPPCRCTNSCPKKPKKP & $\checkmark$ & & \\
\hline STR24_Sx4.1 & A & QKSLVPSVITTCCGYDPGTMCPPCRCTNSC & $\checkmark$ & & \\
\hline STR25 & A & QKSLVPSVITTCCGYDPGTMCPPCRCTNSCKTKPKK & $\checkmark$ & & \\
\hline STR26 & A & OKELVPSVITTCCGYDPGTMCPPCRCTNSCKTKPKK & $\checkmark$ & & \\
\hline STR34 & B2 & $\begin{array}{l}\text { KQHSQFNADENKAAFDSEDSLGNFMDFLHNEKGDKLPFANVDSAATDLGQFQPSAENEDGK- } \\
\text { FRFFDRQQ }\end{array}$ & $\checkmark$ & $\checkmark$ & $\checkmark$ \\
\hline STR36 & B2 & $\begin{array}{l}\text { KQHSQFNADENKAAFDSEDSLGNFMDFLHNEKGDKVPFANVDSAATDLGQFQPSAENEDGK- } \\
\text { FRFFDRQQ }\end{array}$ & $\checkmark$ & $\checkmark$ & $\checkmark$ \\
\hline STR37 & B2 & $\begin{array}{l}\text { KQHSQFNADENKAAFDSEDSLGNFMDFLHNEKGDKVPFANVDSAATDLGQFQPSAENEDGK- } \\
\text { FRFFDRQQ }\end{array}$ & $\checkmark$ & & \\
\hline STR39 & B2 & $\begin{array}{l}\text { KQHSQFNADENKAAFDSEDSLGNFMDFLHNEKGDKLPFANVDSAAADLGQFQPSAENEDGK- } \\
\text { FRFFDRQQ }\end{array}$ & & $\checkmark$ & \\
\hline STR41 & Con-ikot-ikot & $\begin{array}{l}\text { SGPADCCRMKECCTDRVNECLQRYSGREDKFVSTCYQEATLTCGSFNEIVGCCYGYQMCMIR- } \\
\text { VVKPNSLSGAHEACKTVSCGNPCA }\end{array}$ & & $\checkmark$ & \\
\hline STR42_Con-ikot-ikot_SI & Con-ikot-ikot & $\begin{array}{l}\text { SGPADCCRMKECCTDRVNECLQRYSGREDKFVSFCYQEATVTCGSFNEIVGCCYGYQMCMIR- } \\
\text { VVKPNSLSGAHEACKTVSCGNPCA }\end{array}$ & $\checkmark$ & $\checkmark$ & $\checkmark$ \\
\hline STR52 & Conkunitzin & DRPSLCDLPADSGSGTKAEKRIYYNSARKQCLRFDYTGQGGNENNFRRTYDCQRTCLYT & $\checkmark$ & & \\
\hline STR53 & Conkunitzin & PSYCNLPADSGSGTKPEQRIYYNSAKKQCVTFTYNGKGGNGNNFSRTNDCRQTCQYPA & $\checkmark$ & & \\
\hline STR54 & Conkunitzin & $\begin{array}{l}\text { PSYCNLPADSGSGTKPEQRIYYNSAKKQCVTFTYNGKGGNGNNFSRTNDCRQTCQYPLYACIS- } \\
\text { GCRCET }\end{array}$ & $\checkmark$ & & \\
\hline STR55_Conkunitzin-S2 & Conkunitzin & $\begin{array}{l}\text { ARPKDRPSYCNLPADSGSGTKPEQRIYYNSAKKQCVTFTYNGKGGNGNNFSRTNDCRQTC- } \\
\text { QYPV }\end{array}$ & $\checkmark$ & $\checkmark$ & \\
\hline STR56_Conkunitzin-S1 & Conkunitzin & KDRPSLCDLPADSGSGTKAEKRIYYNSARKQCLRFDYTGQGGNENNFRRTYDCQRTCLYT & $\checkmark$ & $\checkmark$ & \\
\hline STR66 & conopressin & CIIRNCPRGGKRDVDETHLTMPCMCCSFRQCGAEYLLWSWRMGNGDRRSDQVH & $\checkmark$ & $\checkmark$ & \\
\hline STR67 & Conophysin & CIIRNCPRGGKRDVDETHLTMPCMCCSFRQCGAPYLLWSWRMGNGDRRSDQVH & & $\checkmark$ & \\
\hline STR70 & G2 & DCQRGCVGCGNRAGCCCGNKYCDKDNTCQEKPAKPST & $\checkmark$ & & \\
\hline STR73 & $\mathrm{H}$ & DSPQSECDGPRCPFICCFYEERKCGTRDCP & & & $\checkmark$ \\
\hline STR76 & I1 & GTCSGVEQQCSNNADCCGELCCLSDKCGSPCMIRL & $\checkmark$ & $\checkmark$ & $\checkmark$ \\
\hline STR105 & M & CCIAPMCRGPCKCCEEPGHP & & $\checkmark$ & \\
\hline STR107_S3-S02 & M & CCPARMCMAACSCCD & & $\checkmark$ & \\
\hline STR115_S3-G04 & M & QKCCGEGSSCPKYFKNNFICGCC & & $\checkmark$ & $\checkmark$ \\
\hline STR116_SIIIB & M & QNCCNGGCSSKWCKGHARCC & & $\checkmark$ & $\checkmark$ \\
\hline STR117_SIIIA & $\mathrm{M}$ & QNCCNGGCSSKWCRDHARCC & & $\checkmark$ & $\checkmark$ \\
\hline STR144_Conotoxin-3 & $\mathrm{O} 1$ & CESYGKPCGIYNDCCNACDPAKKTCT & $\checkmark$ & $\checkmark$ & $\checkmark$ \\
\hline STR146_SO3 & $\mathrm{O} 1$ & CKAAGKPCSRIAYNCCTGSCRSGKC & & $\checkmark$ & $\checkmark$ \\
\hline STR147_S6.1 & $\mathrm{O} 1$ & CKAAGKSCSRIAYNCCTGSCRSGKC & & $\checkmark$ & $\checkmark$ \\
\hline STR148_SVIB & $\mathrm{O} 1$ & CKLKGQSCRKTSYDCCSGSCGRSGKC & $\checkmark$ & $\checkmark$ & \\
\hline STR150 & $\mathrm{O} 1$ & CRPSGSNCGNISICCGRCVNRRCT & & $\checkmark$ & $\checkmark$ \\
\hline STR151_SVIA mutant 1 & $\mathrm{O} 1$ & CRPSGSPCGVTSICCGRCYRGKCT & & $\checkmark$ & $\checkmark$ \\
\hline STR152_SVIA & $\mathrm{O} 1$ & CRSSGSPCGVTSICCGRCYRGKCT & $\checkmark$ & $\checkmark$ & $\checkmark$ \\
\hline STR157 & $\mathrm{O} 1$ & DCGEGGQGCYTRPCCPGRECVAGATGGGVCL & & $\checkmark$ & $\checkmark$ \\
\hline STR183_S6.8 & $\mathrm{O} 1$ & DGCSNAGGFCGIHPGLCCSEICLVWCT & $\checkmark$ & & \\
\hline STR184_SVIE & O1 & DGCSSGGTFCGIHPGLCCSEFCFLWCITFID & & & $\checkmark$ \\
\hline STR186 & $\mathrm{O} 1$ & DKQEYHAVRKWSCVKRGDSCKTNICCAGLTCLRAHAINICLYLMPI & & $\checkmark$ & \\
\hline STR187_Mr022 & $\mathrm{O} 1$ & ECREKGQGCTNTALCCPGLECEGQSQGGLCVDN & & & $\checkmark$ \\
\hline STR207 & $\mathrm{O} 1$ & KSWSCVEHGDSCKTNICCAGLTCLRAHAINLCLYLMPM & & $\checkmark$ & $\checkmark$ \\
\hline STR216-SO4 & $\mathrm{O} 1$ & STTKVSKATDCIEAGNYCGPTVMKICCGFCSPYSKICMNYPKN & & $\checkmark$ & $\checkmark$ \\
\hline STR218_SO5 & $\mathrm{O} 1$ & STTKVSKSTSCMEAGSYCGSTTRICCGYCAYFGKKCIDYPSN & & $\checkmark$ & $\checkmark$ \\
\hline STR221 & $\mathrm{O} 1$ & VRESDSCRKLGERCPSRPCCPRLRCGSGRAGGVCRHPYN & & $\checkmark$ & $\checkmark$ \\
\hline STR222 & $\mathrm{O} 1$ & VRESEGCAGLGAPCRYRRCCRRLKCVGGHVGRACRYPANYYYYY & & $\checkmark$ & $\checkmark$ \\
\hline STR229_contryphan-G & contryphan & GCPWEPWC & & $\checkmark$ & $\checkmark$ \\
\hline STR249 & $\mathrm{O} 2$ & KNCAYAFDACTSDRQCCSGYCVGNVYCE & $\checkmark$ & $\checkmark$ & $\checkmark$ \\
\hline \multicolumn{6}{|l|}{ Continued } \\
\hline
\end{tabular}




\begin{tabular}{|c|c|c|c|c|c|}
\hline Sequence ID & Superfamily & Mature sequence* & Distal & Central & Proximal \\
\hline STR252 & $\mathrm{O} 2$ & KSNAESWWEGECRTWNAPCSFTSQCCFGKCAHHRCIAW & $\checkmark$ & $\checkmark$ & $\checkmark$ \\
\hline STR259 & $\mathrm{O} 2$ & IYYNSARKQCLRFDYTGQGGNENNFRRTYDCQRTCLYT & $\checkmark$ & $\checkmark$ & $\checkmark$ \\
\hline STR297 & $\mathrm{O} 3$ & DKQEYHAVRKWSCVKRGDSCKTNICCAGLTCLRARAINVCLYLMPI & $\checkmark$ & & \\
\hline STR301 & O3 & NTPDDGTCKSSSNCSTGQTCCKANAKNEKGFCTEDCWF & & $\checkmark$ & \\
\hline STR303 & $\mathrm{O} 3$ & TADEACKEYCEERNKNCCGRTNGEPRCASMCF & & $\checkmark$ & $\checkmark$ \\
\hline STR312 & O3 & TVDEECKEYCEQRNKNCCGETNGEPVCAQACL & & $\checkmark$ & $\checkmark$ \\
\hline STR320 & $\mathrm{P}$ & RVNCAGTLCQNGKCGGDCICRPANSTHQDCQPNDFD & & $\checkmark$ & $\checkmark$ \\
\hline STR321 & $\mathrm{P} / \mathrm{O} 2$ & KSNAESWWEGECRTWNAPCSSTSQCCFGRCAHHRCIAW & & $\checkmark$ & \\
\hline STR326 & Sf-Mi2 & DCQRGCVGCGNRAGCCCGNKYCDKDNTCQPNPVWL & $\checkmark$ & $\checkmark$ & $\checkmark$ \\
\hline STR333 & $\mathrm{T}$ & HCCPIDLPCCPL & & $\checkmark$ & \\
\hline STR337 & $\mathrm{W}$ & ISKSMGDVVGRTWWCPPEGELTHAGSATKQLLSSVWGLIGGVLRMLDQNRRH & $\checkmark$ & $\checkmark$ & $\checkmark$ \\
\hline
\end{tabular}

Table 1. Integration of the transcriptome and proteomic data using ProteinPilot tool. Underlined sequences are matched to $99 \%$ confidence (list of peptide fragments are shown in supplementary table S8) to the proteomic data obtained from the three venom duct sections of $C$. striatus. MS/MS fragments obtained from reduced-alkylated and reduced-alkylated-trypsin digested extracted venom from three venom duct sections of specimens A, B and C of C. striatus were used to map their presence in the proteome.

(a)
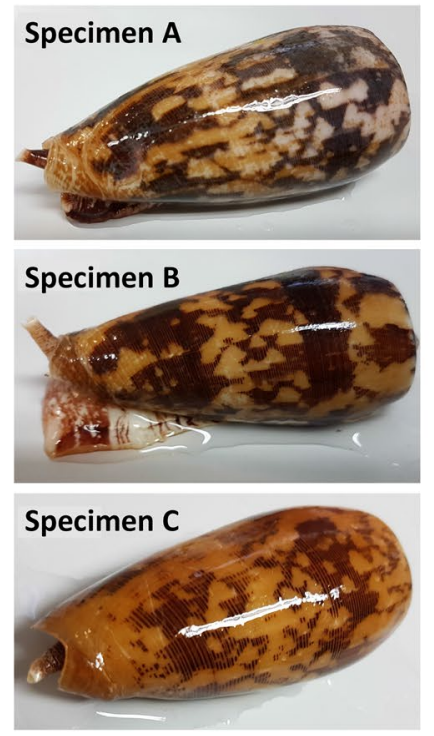

(b)

\section{Total 370 Transcripts (Tr) \\ 25 Superfamilies (SF)}

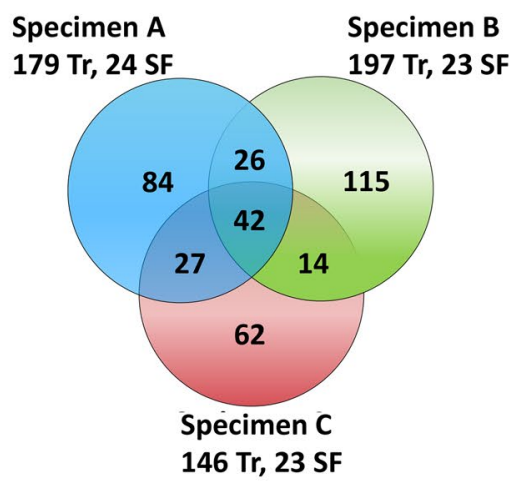

(c)

(d)
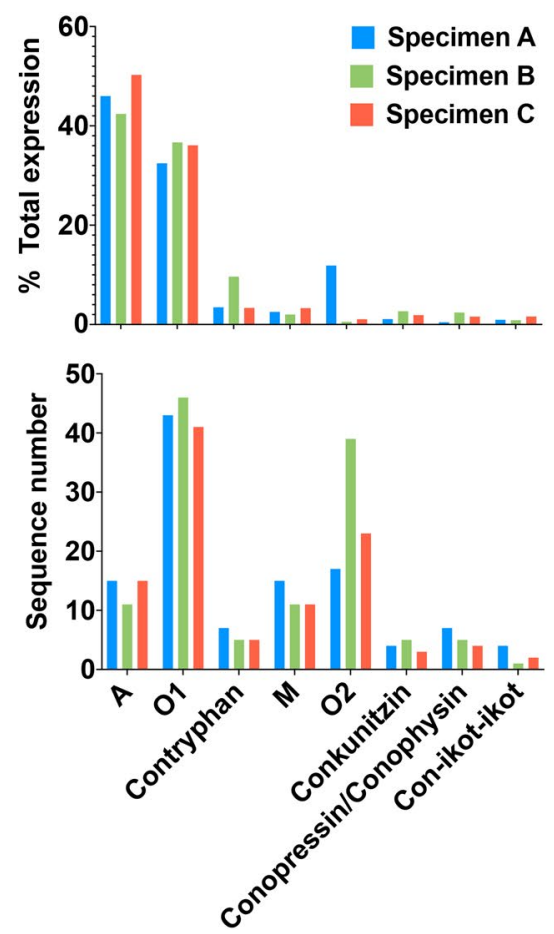

Figure 1. Intraspecific venom transcript variation in between three adult C. striatus (specimens A-C). (a) Photographs of the three specimens of adult C. striatus used in the study. (b) Venn diagram showing the common and unique conotoxin precursor transcripts among the three specimens of C. striatus. (c) Expression levels of the dominant conotoxin gene superfamilies across the three specimens. Relative expression levels were calculated for each superfamily using the TPM values. (d) The number of conotoxin precursors found in the dominant gene superfamilies.

third largest superfamily identified in C. striatus transcriptomes, with highest expression in proximal sections, among to other fish hunters studied to-date contryphans were found to be dominant in the Atlantic cone snail C. ermineus (Chelyconus) ${ }^{9}$, where again higher expression levels were seen proximally. The M superfamily was also highly diverse, with $24 \mu$-like conotoxins as well as four cysteine poor precursors related to the unclassified two-cysteine Ec2C01 from C. emaciatus. The expression levels of $\mu$-like conotoxins also increased proximally (Fig. 2A) while the expression levels of cysteine poor peptides remained low along the venom duct. Among the $24 \mathrm{M}$ superfamily conotoxin precursors with framework III, nine previously described $\mu$-conotoxins and/or $\mu$-like conotoxins were identified. However, SIIIB and S3-GO4 were the only dominant $\mu$-conotoxins across all three specimens (Fig. 3, supplementary table S3). 
(a)
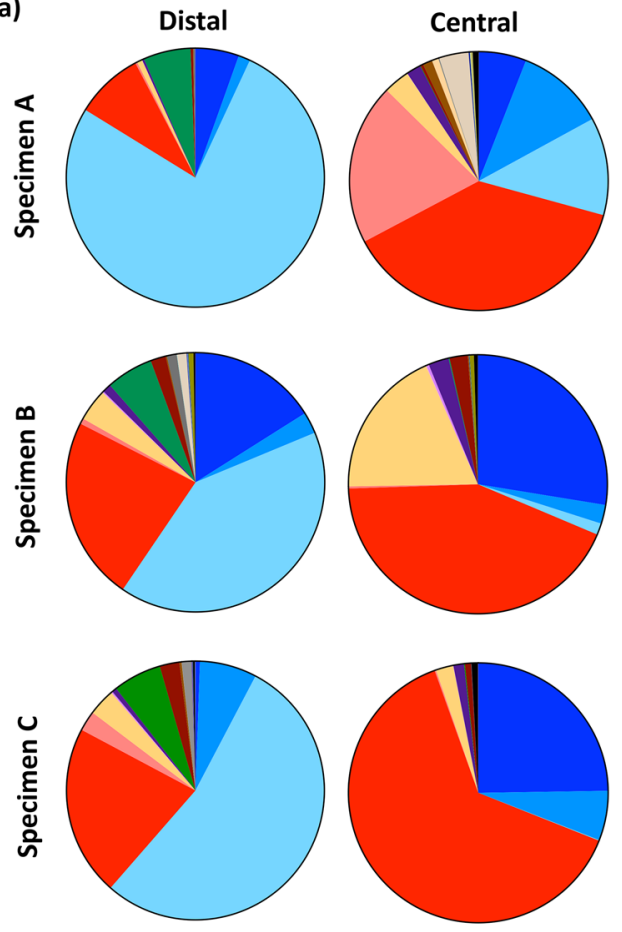
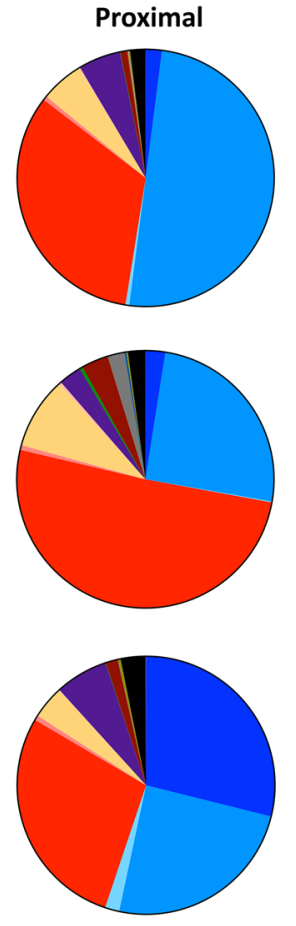

(b)

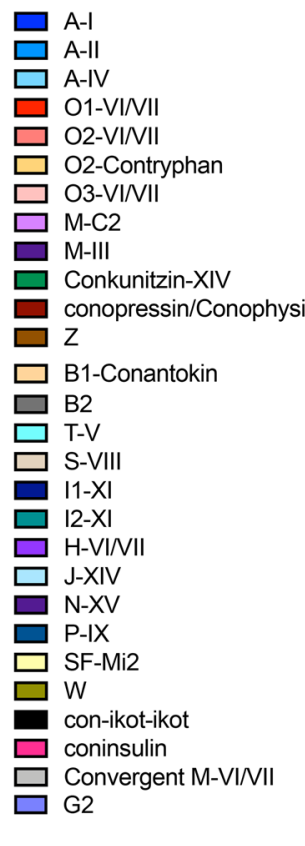

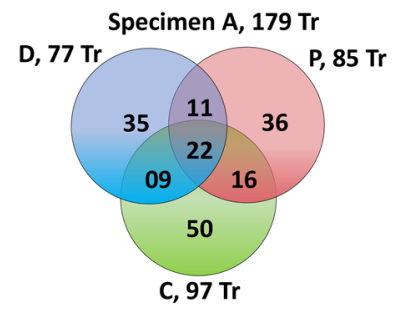

Specimen B, $194 \mathrm{Tr}$

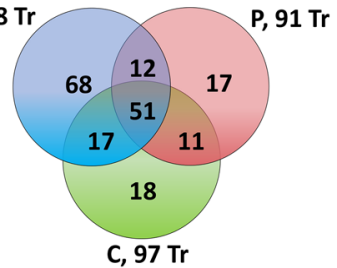

Specimen C, $146 \mathrm{Tr}$

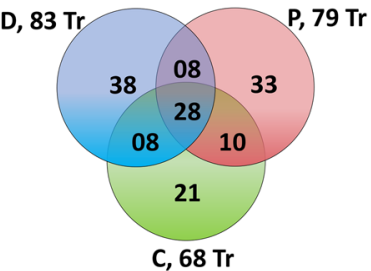

Figure 2. Comparison of the transcriptomic profiles of three venom duct sections (distal, central and proximal) obtained from three adult C. striatus (specimens A-C). (a) Venn diagram showing the common and unique conotoxin precursor transcripts among the three venom duct sections in three specimens of C. striatus. (b) Expression profiles (Relative TPM) of all identified superfamilies across the venom duct sections in three specimens of $C$. striatus. $T r$ transcript, $D$ distal-blue, $C$ central-green, $P$ proximal-red.

Highly abundant conotoxin precursors are conserved across individual C. striatus. When the conotoxin precursor profiles were compared across the duct sections of specimens A-C, only $12 \%, 25 \%$ and $19 \%$ of venom peptides were common to all three sections, with $32 \%, 48 \%$ and $36 \%$ shared between at least two duct segments, respectively (Fig. 2B). 45 of 58 (A), 74 of 91 (B) and 43 of 54 (C) of the shared precursor transcripts belonged to the dominant superfamilies O1, A-I, A-II, O2 and M. However, of the 370 conotoxin precursors found in specimens A-C, only 61 were major transcripts ( $>1 \%$ of maximum TPM), including 48 belonging to these seven dominant superfamilies (Fig. 3A,B). Interestingly, most of these highly abundant transcripts were expressed along the full length of the venom duct (Fig. 3A). In contrast, of the unique peptides found in each specimen (Fig. 2B), only 9 of 121 (STRA), 11 of 103 (STRB) and 8 of 92 (STRC) were highly expressing major transcripts. This finding reveals that minor transcripts ( $<1 \%$ of maximum TPM) are disproportionate drivers of intraspecific variability, while highly expressed peptides are more conserved albeit with variable levels of expression along the venom duct (Fig. 3A). When the dominant and the minor peptides are compared across the major superfamilies, it is evident that superfamilies A-I, O1, O2, M, and contryphan contribute most to this variability, with a high number of minor peptides (155) compared to the A-II, A-IV, conkunitzin and con-ikot-ikot superfamilies that have fewer minor peptides (seven) across all specimens (Fig. 3C).

Proteomic variability. LC-MS/MS data of the reduced alkylated and trypsin digested injected and extracted venoms from the trisected venom ducts of specimens A-C were searched against the respective conotoxin transcriptomic data to match 62 mature conotoxins belonging to 17 superfamilies in ProteinPilot (confidence value $>99 \%$ ) (Table 1). Thirty three (54\%) of these matched conotoxins were highly expressed in the transcriptome (Fig. 3), including 36 that were novel and currently uncharacterised. Typically proteomes of cone snails are 10-50-fold more diverse than their matching transcriptomes due to variable peptide processing and variable post translational modifications ${ }^{29}$. This proteomic complexity found in the relatively recently evolved cone snail venoms ${ }^{16,25-27,29}$ exceeds the diversity of venoms in spiders, wasps and centipedes ${ }^{21}$, especially when intraspecific variability is considered. Considering only the peptide monoisotopic masses ((all mentions of masses are monoisotopic unless specified otherwise) between $800-10,000 \mathrm{Da}$ in extracted C. striatus venom duct sections the total unique masses detected were 10,799, 9665 and 10,306 in specimens A-C, respectively (Fig. 4A), consistent with the high level of diversity observed previously in cone snails ${ }^{4,7,16,23,24}$. Impressively, most peptide masses were unique to each specimen despite their common superfamily origins and between the three specimens a total of 18,347 unique peptide masses were detected (at a mass precision of $0.25 \mathrm{Da}$ ). Intraspecific venom duct peptide mass diversity was also high between comparable venom duct sections across the three specimens, with only $22.7 \%, 28.3 \%$ and $26.6 \%$ shared between at least two specimens in the distal, central and proximal duct sections, respectively (Fig. 4B). Proteomic data for each duct sections revealed 2-, 2.4- and 
(a)

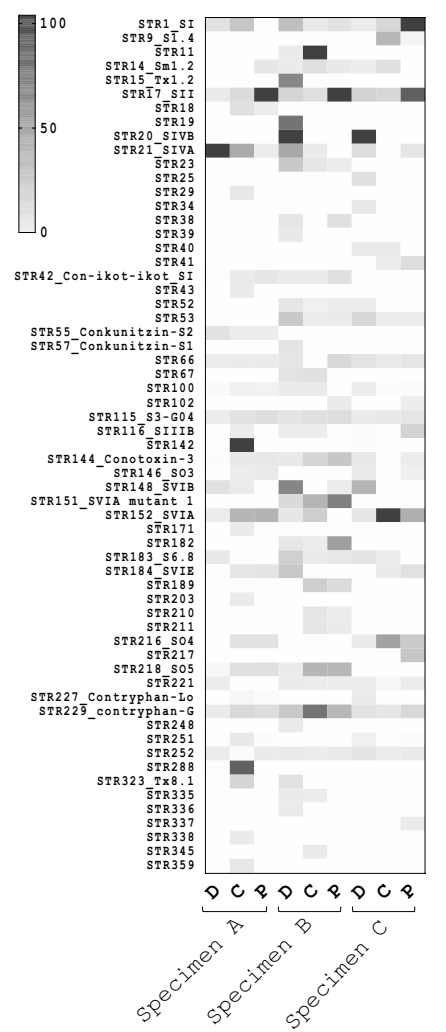

(b)

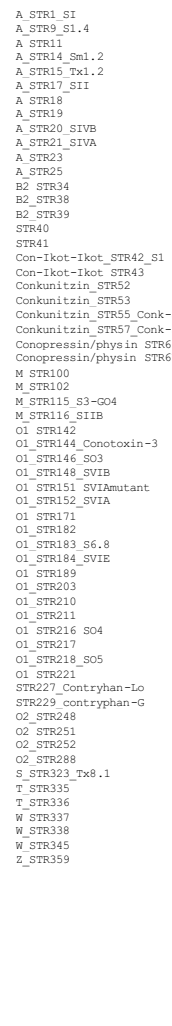

(c)
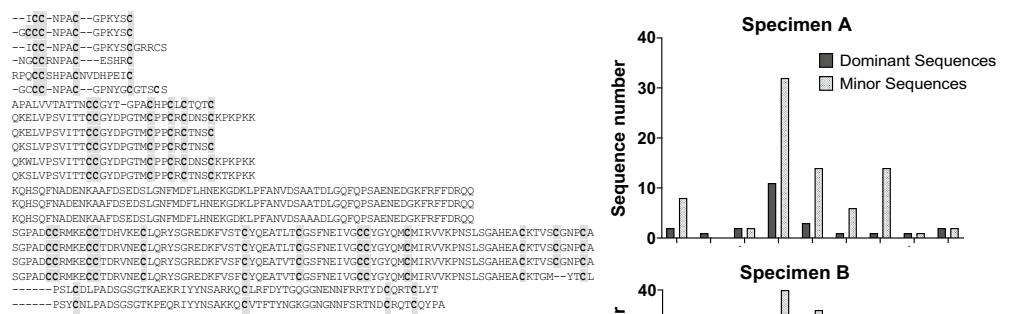

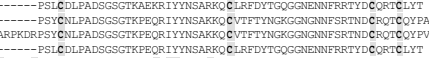

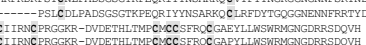

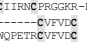

OQPETRCVFVDC
OKCGEGSSCKYEKN-NFTCGCC
ONCCNGG-CSSE-KNCKGHARCC

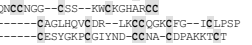

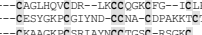

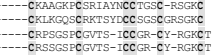

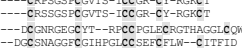

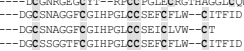

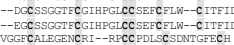

GGECALEGENCRI--RPCC PDLSCS

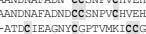

-ATDC IEAGNYCGPTVMKICCGECS SPSSKCC MNYPFN

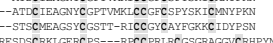

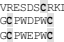

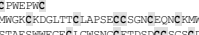

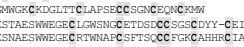

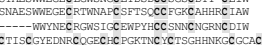

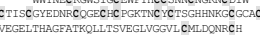

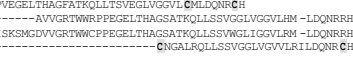

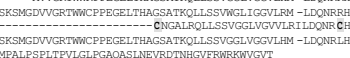

Specimen B
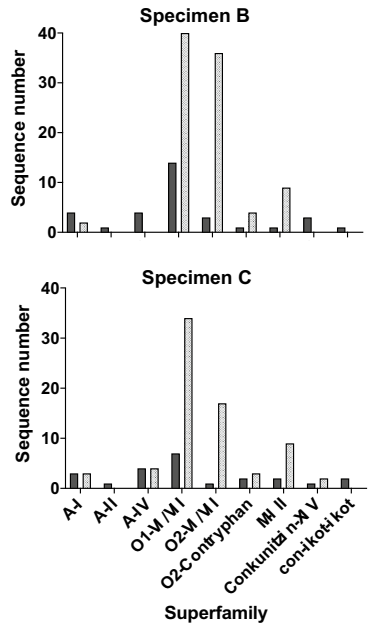

Figure 3. Expression level variation of the major conotoxin (relative expression level $>1 \%$ ) precursors across the transcriptomes of three venom duct sections (distal, central and proximal) in three adult C. striatus (specimens A-C). (a) Heat map showing the distribution of dominant peptides across the duct section in three specimens. Relative expression levels are indicated as \% TPM relative to the maximum TPM in each venom duct segment transcriptome. (b) The mature conotoxin sequence of the dominant sequences found across all nine transcriptomes studied in the same rank order as the heat map. (c) Bar graphs visualizing the contribution of the dominant and minor peptides to each main superfamily in three specimens of C. striatus.

8.7-fold higher numbers of peptide masses in the proximal section of the venom duct compared to the distal in specimens A-C, respectively (Fig. 4A, supplementary Figure S2). In contrast, the central section comprised higher number of shared peptide masses with the proximal duct and fewer with distal venom duct (Fig. 4A). Overall, this profiles reflected in the conotoxin expression profiles at the transcriptomic level. (Fig. 2A).

The variability of the peptide expression patterns in the segmented venom duct LC-MS profiles (native (native undigested extracted venoms) across three specimens was statistically analysed using a supervised PCA (PC1 $50 \%$ and PC2 50\%). Visualised results of the PCA showed a clear differentiation between mass profiles of the three venom duct segments (Fig. 4C), while matching duct sections of each specimen clustered closely together. Localised conotoxin superfamilies and variable expression of minor peptides across and among the venom duct section may have contributed to the distances within and between these clusters. For example, distances between the distal segments were relatively high, indicative of higher levels of variability in expressed peptides in contrast to the central and proximal which clustered more tightly. Earlier findings revealed that the distal venom duct produced the predatory venom in C. geographus and C. marmoreus ${ }^{4}$. We propose that the high level of venom diversification seen distally in C. striatus may reflect its more recent origins and associated stronger diversifying selection pressures associated with the diet shift to fish hunting ${ }^{4,8}$.

Venom duct origins and biochemistry of predatory and defensive venoms. Ancestral wormhunting cone snails are hypothesized to have repurposed defensive venoms used against predatory fish and other molluscs to facilitate prey diversification to mollusc- and fish-hunting ${ }^{4,8}$. The defensive venoms of worm hunters studied so far is originated in the proximal part of the venom duct ${ }^{5,6}$. However, the venom duct origins of the predatory and defensive venoms of hook and line fish hunting cone snails belonging to the Pionoconus clade has not been defined. Although collecting predatory venom from C. striatus is straightforward using fish as a stimulant, obtaining defensive venom has not previously been achieved. After repeated attempts on 9 specimens, we successfully collected defensive venom from C. striatus specimen D (supplementary Figure S3). MALDI spot imaging analysis was used to visualise the correlation of expressed peptide masses in injected predatory and defensive venoms to the extracted venoms from 8 venom duct segments from the same specimen (Fig. 5A). This analysis revealed that peptide expression profile of distal (Sections 1,2 and 3) and proximal (Sections 6,7 and 
(a)

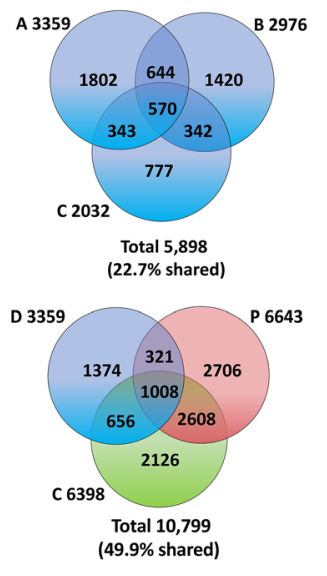

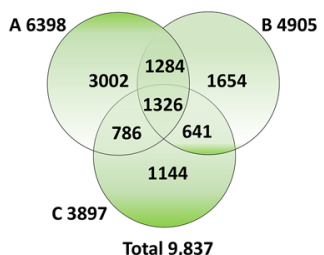
(26.6\% shared)

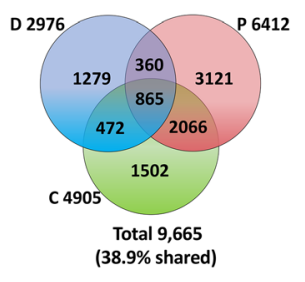

Central venom duct

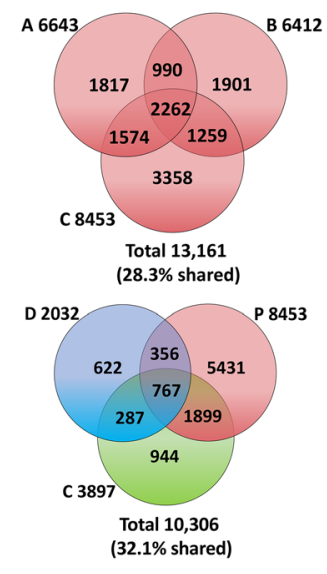

Proximal venom duct (c)

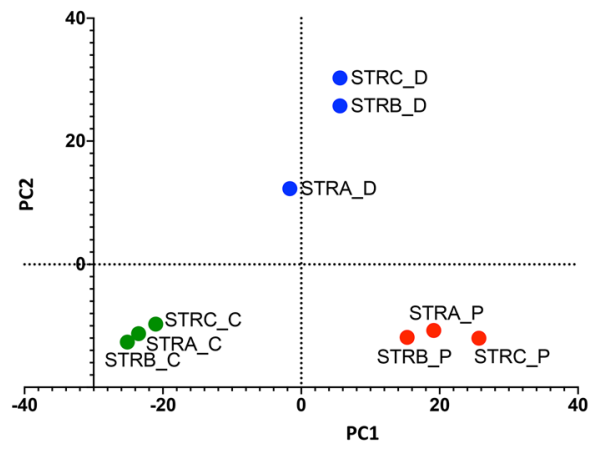

Figure 4. Comparison of the peptide distribution in three venom duct sections (distal, central and proximal) across the three adult $C$. striatus (specimens A-C). (a) Venn diagrams showing the common and unique peptide masses across the three venom duct sections (D, C and $\mathrm{P}$ ) in three specimens (A, B and C) of C. striatus. Total mass units detected in the LC-MS for each specimen is shown below the respective Venn diagram. Total mass units detected in the LC-MS for each specimen is shown below the respective Venn diagram. (b) Venn diagrams showing the mass variability in each venom duct section $(D, C$ and $P)$ across the three specimens (A, $\mathrm{B}$ and C) of C. striatus. Percentage of the shared masses between the specimens are shown under the each Venn diagram. (c) PCA plot showing the variability of the venom extracted from dissected venom duct sections in three specimens. LC-MS data generated from SCIEX 5600 QTOF instrument is statistically analysed using the MarkerView software to obtain the PCA and loading plots.

8) sections were distinct, while the central segments (Sections 4 and 5) shared peptide masses from both ends of the venom duct (Fig. 5A) supporting the proteomic and transcriptomic results of distal, central and proximal segments of specimens A, B and C reported above (Figs. 3A and $4 \mathrm{~A}$ ). The injected venoms and the venom duct extracts from specimen D were further analysed using Triple TOF LC/MS/MS. The expression levels of 40 most abundant peptide masses expressed across the samples were chosen to define the venom duct origins of predatory and defensive venoms. Eighteen of these 40 abundant masses were matched to highly expressed precursors in the transcriptome (Figs. 3A and 5B). However, despite their abundance, confident comparative sequence annotation for the remaining 22 abundant masses was not possible, likely due to high levels of post translational modification obscuring their transcriptomic origins.

MALDI mass imaging data and the Triple-TOF LC/MS/MS data revealed clear links between venom duct localisation and function, with most defensive venom peptides arising proximally and most predatory venom peptides arising distally, reminiscent of the pattern found in C. geographus and C. marmoreous ${ }^{4}$. The major venom components of the proximal venom duct included $\omega$-conotoxins SVIA, conotoxin-3, SO4 and SO5, $\delta$-conotoxin SVIE, $\alpha$-conotoxins SI and SII, $\mu$-conotoxin S3-GO4, con-ikot-ikot S, and conopressin/conophysin STR67 (Fig. 5B). Consistent with previous observations, these conotoxins were also highly expressed in the defensive venom (Fig. 5C) and were only present in the predatory venom at lower levels or were not detected (Fig. 5A,C). On the other hand, conkunitzin S1, conkunitzin S2 and STR54, and $\omega$-conotoxin SVIB were more prominent in the predatory venom (Fig. 5C) and readily identified in distal duct segments (Fig. 5B). Interest-

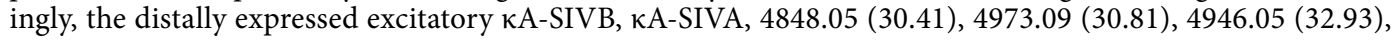
4752.74 (31.85) and 3840.44 (36.69) were the most abundant $\kappa A$-conotoxins/ $\kappa A$-like glycosylated peptides in both the predatory and defensive venoms (Fig. 5C). This suggest a differential regulation of peptide expression along the venom duct can occur at the molecular level to explain its ability to be deployed in both modes of envenomation as key components. Mature $\kappa \mathrm{A}$-conotoxins are O-linked glycosylated at positions 7 (serine/ tyrosine) and/or 9 (tyrosine $)^{30}$, therefore, glycan groups (NexNAc + and Hex-HexNAc +) were screened in the injected venom proteomes to identify probable $\kappa$ A related peptides (Supplementary figure S4 and S5). SIVB, 4848.05 (30.41), 4973.09 (30.81), 4946.05 (32.93), 4752.74 (31.85) and 3840.44 (36.69) and SIVA were the most abundant $\kappa \mathrm{A}$-conotoxins in both the predatory and defensive venoms (Fig. $5 \mathrm{C}$ ) despite only being expressed in the distal venom duct sections (Fig. 5A,C).

KA-conotoxins as evolutionary driver for the successful radiation of the Pionoconus group? The biochemical innovation of achieving an immediate immobility of the agile prey is suggested to be one of the driving forces of origin and success piscivory ${ }^{10,16}$. Excitatory $\delta$-conotoxins found in fish hunting cone snail venoms that initiate rapid onset tetanic paralysis of the prey ${ }^{16}$ are believed to have underpinned the successful dietary switch to piscivory $y^{31,32}$ via repurposing the defensive venom of ancestral worm hunting species s $^{33}$. Despite the presence of $\delta$-conotoxins, $\kappa \mathrm{A}$-conotoxins are the major class of excitatory conotoxins identified to date in the predatory venom of Pionoconus cone snails C. catus $^{16}$, C. consors $^{34,35}$ and C. striatus ${ }^{35}$, C. striolatus ${ }^{36}$ and C. magus $^{17}$ reflecting the importance of their excitatory mode of action. Indeed, injection of a C. catus preda- 
(a)

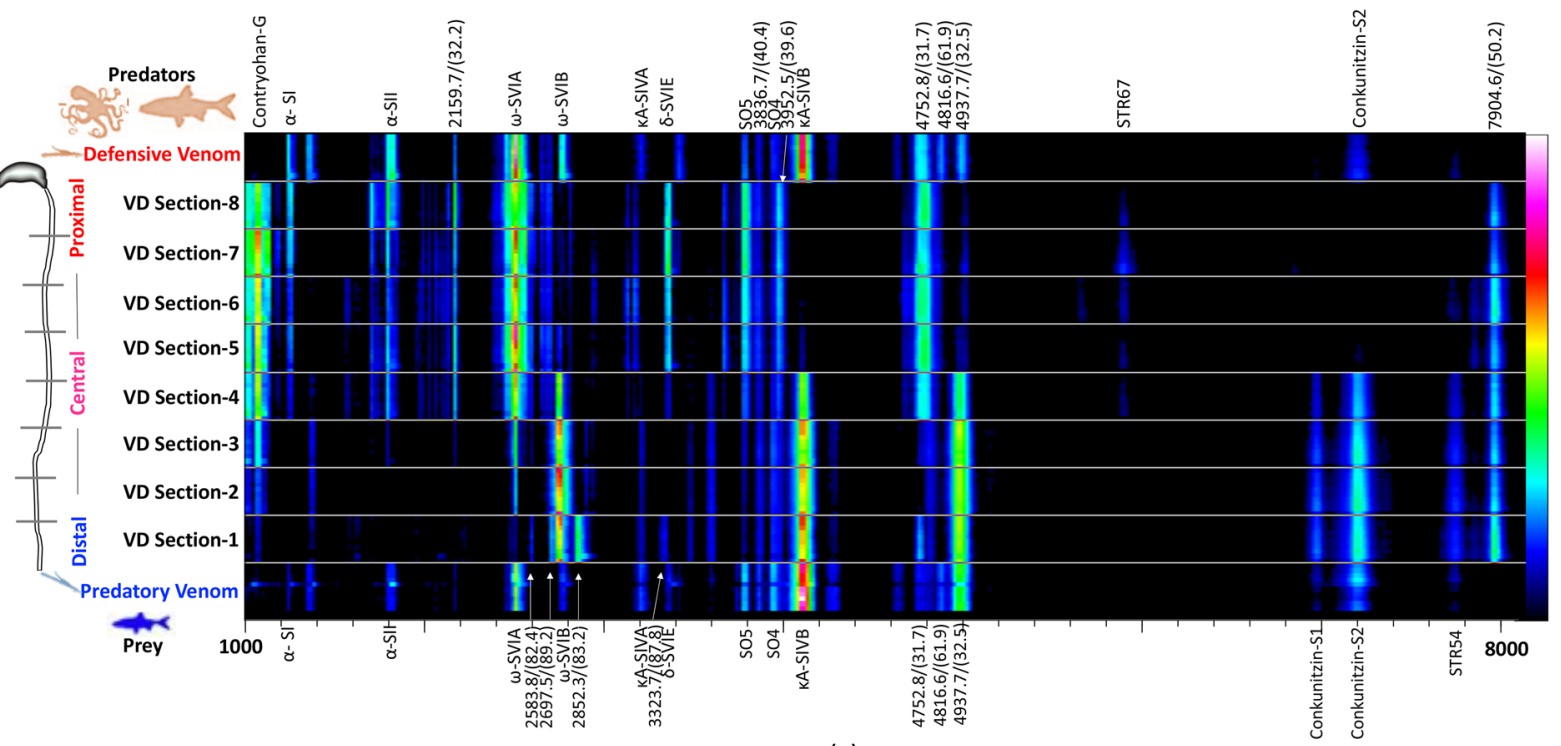

(b)

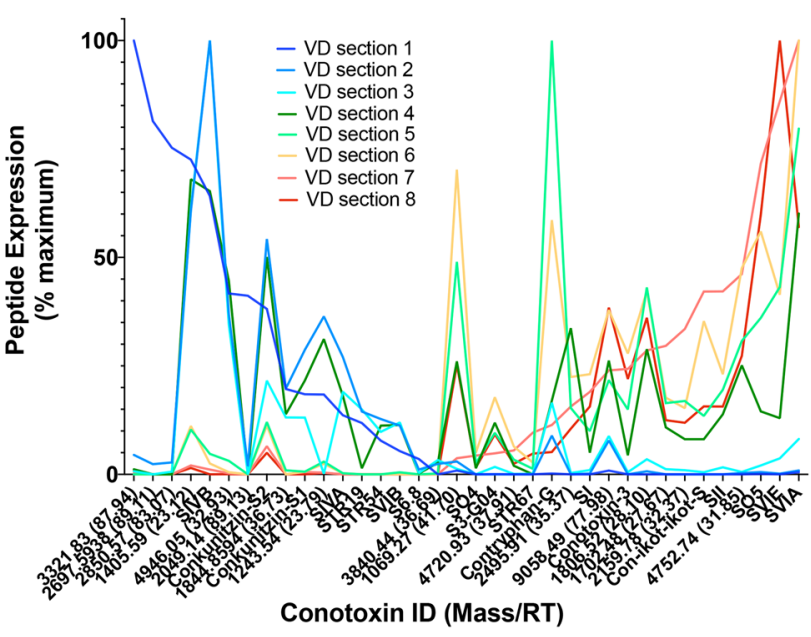

(c)

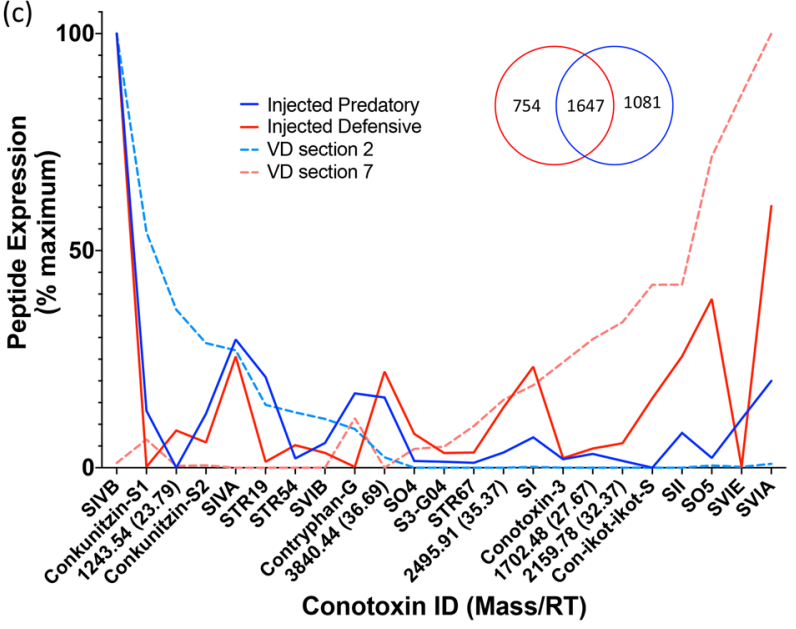

Figure 5. Visualisation of the venom distribution pattern across eight venom duct sections and their correlation to the injected predatory and defensive venoms obtained from the same specimen of C. striatus (specimen D). (a) MALDI spot imaging showing the alignment of the injected predatory and defensive venoms with extracted venoms of eight venom duct sections of the injected and extracted venoms. (b) Quantitative analysis of the major peptides found in the LC/MS profiles of the dissected venoms (venoms extracted from 8 duct segments). Both the retention time and the mass was considered when identifying the peptides across the duct sections and injected venoms. Peptides are ranked to the expression levels (relative to the maximum intensity of each sample) of section 1 (left) and 7 (right). (c) Mass profile comparisons were made between the LC/MS data obtained by injecting similar amount $(1 \mu \mathrm{l})$ of the injected predatory and defensive venoms in comparison the distal duct section 2 and proximal duct section 7. Peptides are ranked by expression level (relative to the maximum intensity of each sample) of section 2 (left) and 7 (right). The inset Venn diagram shows the unique and the shared masses in each venom. Reconstructed LC/MS chromatograms were used to analyse the mass profiles and their expression levels. Figure 5b,c are also shown in heat map versions in supplementary Figure S6.

tory venom derived mixture of $\mathrm{\kappa A}$-conotoxins $\left(\mathrm{EC}_{50}\right.$ of $\left.34.74 \mathrm{ng} / \mathrm{g}\right)$ was found to be effective in initiating tetanic paralysis in zebra fish ${ }^{16}$.

Given the important role of $\kappa$ A-conotoxins in the venoms of Pinoconus clade fish hunters, we explored their diversity across the fish hunting cone snail lineages. Previous phylogenetic evidence suggests that multiple independent diversification events define the evolution of mollusc hunting (Calibanus, Cylinder, Conus, Dariconus, Eugeniconus and Leptoconus) and fish hunting (Phasmoconus, Gastridium, Pionoconus, Textilia, Afonsoconus, Embrikena, Asprella and Chelyconus) lineages from ancestral worm hunters ${ }^{12,13,37}$. This evolutionary diversification was underpinned by shifts in venom chemistry, radula morphology and prey capture behaviours facilitating these significant dietary shifts. Among these new lineages, the hook and line fish hunters are the most successful in-terms of species number and distribution ${ }^{12}$, with the Pionoconus clade being the most successful with 40 known fish hunting species (supplementary Table S7).

When the available venom compositions of the representative species of the fish hunting clades are compared, кA-conotoxins are more conserved in the Pionoconus clade (Table 2). Three main groups have been identified 


\section{Full length $\mathrm{KA-conotoxins}$}

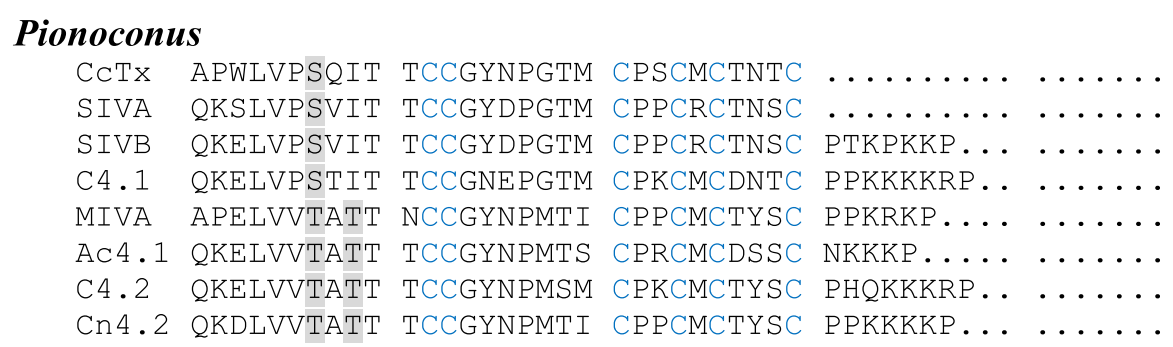

\section{Textilia}

Bu27 APELVVTATt TCCGYDPMTI CPPCMCTHSC PPKRKP.........

\section{Short KA-conotoxins}

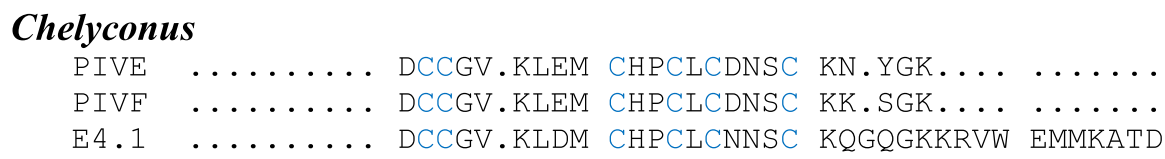

\section{$\alpha$ A-conotoxins}

\section{Chelyconus}

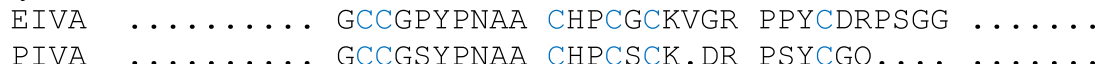

\section{Embrikena}
PEIVA ........ . CCGV.PNAA CHPCVCTGKC

PeIVB ........ CCGI. PNAA CHPCVCTGKC ...........

\section{Gastridium}

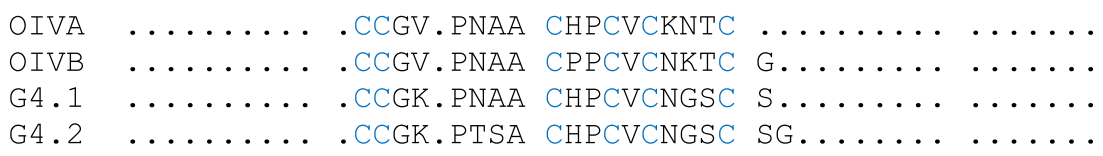

Table 2. Complexity and the distribution of the A superfamily Cysteine framework IV peptides across fish hunting clades of cone snails.

based on the mature peptide sequence; full length $\kappa \mathrm{A}$-conotoxins with a conserved serine at the position 7 , full length $\kappa \mathrm{A}$-conotoxins with a conserved tyrosine at the positions 7 and 9 , and relatively short $\kappa \mathrm{A}$-conotoxins. $\mathrm{Bu} 27$ from the C. bullatus (Textila) transcriptome is the only full length $\kappa \mathrm{A}$-conotoxin (tyrosine at the positions 7 and 9) identified outside the Pionoconus clade ${ }^{38}$. Interestingly the Pionoconus and Textila are sister clades ${ }^{12}$, while the short excitatory $\kappa \mathrm{A}$-conotoxins have only been reported in Atlantic fish hunting C. ermineus ${ }^{9}$ and $C$. purpuracens $^{39}$ of the distinct Chelyconus clade (Table 2). Long $\mathrm{\kappa A-conotoxins} \mathrm{are} \mathrm{also} \mathrm{not} \mathrm{found} \mathrm{in} \mathrm{net} \mathrm{hunters}$ (C. geographus and C. tulipa) and other linages of suggested fish hunters (Phasmoconus, Afonsoconus, Embrikena and Asprella), or mollusc hunting or worm hunting clades investigated to-date, suggesting they have evolved relatively recently.

Most A superfamily peptides have two disulphide bonds and target nicotinic receptors ${ }^{2,40}$. Through gene duplication and positive selection, the A superfamily successfully incorporated a third disulphide bond seen in framework IV aA-conotoxins and $\kappa \mathrm{A}$-conotoxins (Table 2). aA-conotoxins that target muscular $\mathrm{nAChRs}$ have been found in C. ermineus (Chelyconus) ${ }^{9}$, C. purpuracens (Chelyconus) ${ }^{41}$, C. pergrandis (Embrikena $)^{42}$, C. geographus (Gastridium) $)^{42}$ and C. obscurus (Gastridium) $)^{43}$. However, no aA-conotoxins have been identified in cone snails of the Pionoconus clade where $\kappa \mathrm{A}$-conotoxins dominate. This clear separation in the presence of $\alpha \mathrm{A}$ and $\kappa A$ conotoxins in different lineages of fish hunters (Table 2) likely reflects distinct evolutionary pressures associated with the dietary shift to fish hunting in cone snails with different hunting strategies ${ }^{44}$. Short excitatory $\kappa \mathrm{A}$ conotoxins found in fish hunting Chelyconus species with structural similarity to aA-conotoxins may present an example of early functional divergence from paralytic to excitatory peptides. 


\section{Conclusions}

This study reports the first characterisation of venom composition across the venom duct of $C$. striatus and its correlation to the respective injected predatory and defensive venoms. A clear venom duct compartmentalisation was observed at both the transcriptomic and proteomic level with peptides belonging to neuromuscular inhibitors of $\mathrm{A}, \mathrm{O} 1$ and $\mathrm{M}$ are highly expressed in the proximal region compared to the distal region and excitatory peptides ( $\kappa A$ and conkunitzins) are distally dominant. This study revealed that these major superfamily conotoxins dominated the C. striatus venom duct are thus likely to be critical for survival and appear to be subjected to purifying selection. In contrast, minor superfamilies were disproportionately more variable and thus less likely to be critical for survival and appear to be subjected to diversifying selection.

Having KA-conotoxins exclusively produced in the distal segment suggest that during adaptation to fish hunting, toxin classes optimised for fish hunting have supplanted predatory worm hunting toxins in the distal venom duct section, perhaps through repurposing of defensive venoms through adaptive predator-prey evolutionary mechanisms as previously explained for other venomous animals ${ }^{45,46}$. This hypothesis can also be adapted to net feeding fish hunters C. geographus and C. tulipa where the distal venom duct sections are enriched with components used for net feeding ${ }^{4,26}$. In contrast, Pionoconus clade cone snails uniquely express high level of $\kappa \mathrm{A}$-conotoxins to support their hook and line hunting behaviour. Based on their expression pattern and mode of action, we propose that $\kappa \mathrm{A}$-conotoxins are the key evolutionary innovation underpinning the explosive adaptive radiation seen for Pionoconus clade fish hunters. Our findings highlight the need to study additional clades of cone snails to determine how the spatial distribution of conotoxins along the venom duct correlate with injected predatory and defensive venoms. Further studies on understanding the molecular mechanisms, gene structures and hypermutation events contributing to divergence events are required to better understand molecular evolution trajectories in these venomous animals.

\section{Methods}

Venom collection. Four adult specimens of C. striatus collected (at the same time) from Coral Reef in the Northern Great Barrier Reef, Australia were used for the study. Snails were housed in marine aquarium at $24-28{ }^{\circ} \mathrm{C}$ and a 12:12 light-dark cycle. Milked predatory venoms were collected from all specimens using cadaver zebrafish as a stimulant and lyophilized and stored at $-20^{\circ} \mathrm{C}$ until use. Three specimens (A, B and C) (Fig. 1D) were sacrificed and the distal (D), central (C) and proximal (P) thirds stripped of cellular content and apportioned for RNA extraction (75\%) and proteomic (25\%) studies. Injected defensive venom from specimen $\mathrm{D}$ was collected by inducing defensive behaviour using a combination of chemical (using a predatory cone snail C. textile) and physical (pushing the foot using a blunt forceps) stimuli, as previously described ${ }^{4}$, whereas the other specimens were unresponsive. Specimen D was scarified 1 week after the last milking and the venom duct dissected into 8 equal portions and the cellular contents stripped for proteomic analysis.

Venom extraction from dissected venom ducts. In house optimised method for conotoxin extraction from dissected cone snail venom ducts was used. Stripped venom duct cells from each section were triturated with $500 \mu \mathrm{l}$ chilled $30 \%$ acetonitrile (ACN) containing $1 \%$ formic acid (FA) and centrifuged for $20 \mathrm{~min}$ at $12,000 \times g$. The supernatant was removed and spun at $12,000 \times g$ for another $10 \mathrm{~min}$ to separate the fine insoluble material from the crude venom. Protein estimates were obtained using NanoDrop (Thermo Fisher Scientific) A280 method. The venom extracts were immediately lyophilised until further use.

RNA extraction and transcriptomic sequencing. To extract total RNA, venom duct cells collected as described above were placed in a $1.5 \mathrm{~mL}$ tube containing $0.5 \mathrm{~mL}$ of TRIzol reagent (Invitrogen) and total RNA extracted following the manufacturer's instructions to yield 5-10 $\mu \mathrm{g}$ of purified total RNA from each section (Table 1). Sequencing libraries were prepared from total RNA using the TruSeq Stranded mRNA Sample Prep Kit with TruSeq library indices (Illumina Inc., San Diego, CA, USA) following the manufacturer's instructions. The sequencing libraries obtained were pooled and sequenced on a $2 \times 150$ bp High Output Kit v2 run on a NextSeq 500 machine (Illumina Inc., San Diego, CA, USA). The acquired transcriptomic data was processed using BBMap tools (version 38.00 from https://jgi.doe.gov/data-and-tools/bbtools/) to remove sequencing adaptors, low quality reads (Phred score $<28$ ), and the reads of $<50$ bases (bbduck.sh in $1=\mathrm{R} 1$.fastq in $2=\mathrm{R} 2$. fastq out $1=$ outputR1. fastq out $2=$ outputR2.fastq $\mathrm{ref}=$ seqadaptorslist.fa qtrim $=\mathrm{rl}$ trimq $=28 \mathrm{ftl}=10$ minlen $=50$ ). The filtered datasets from each section were then merged as paired end reads using BBMap tools (bbmerge.sh in 1 = outputR1.fastq in $2=$ outputR 2 .fastq out $=$ merged.fastq outu $1=$ unmerged_outputR1.fastq outu2 = unmerged_outputR2.fastq) and then the merged and unmerged reads were assembled as a single reads using Trinity-v2.8.4 (https://github. com/trinityrnaseq/trinityrnase) using a kmer size of -19 and -31 , a maximum chrysalis cluster size of 40 , with no Butterfly transcript reduction parameters set to better identify low level transcripts and transcript variants as described before ${ }^{36}$. The assembled transcripts from both kmers were merged in a single dataset and the duplicate transcripts were removed using BBMap tools (dedupe.sh in = merged_assembled_transcripts.fasta out $=$ duplicates_removed_transcripts.fasta). The candidate conotoxins and conopeptides were identified as previously described $^{47}$ using the latest available versions of the software. After ConoSorter analysis, sequences with signal peptides were retrieved by the signalP4.1 server (http://www.cbs.dtu.dk/services/SignalP-4.1/) using default parameters. The retrieved sequences were submitted to BLASTp (blast +version 2.4.0, e-value $=0.75$ ) against the non-redundant UniProt database before classifying the sequences into superfamily. To classify the sequences into the superfamilies, the sequences were classified based on their signal sequences using cd-hit-v4.6.7 (https:// github.com/weizhongli/cdhit/releases/tag/V4.6.7) with signal sequence identity $>75 \%$. Finally, the annotated conotoxin transcripts were quantified using salmon $v 0.11 .3$ (https://github.com/COMBINE-lab/salmon) with 
default parameter settings. All the computational analyses were performed in high performance computing cluster in PBS Pro environment.

ConoPrec software (http://www.conoserver.org) was then used to identify the conserved signal sequences, cysteine frameworks, cleavage sites and previously reported conotoxins ${ }^{48}$. During this process, precursors $<40$ amino acids in length, signal sequence hydrophobicity less than $40 \%$, and repeated sequences were manually removed. Considering the published variations in signal conservation within superfamilies, the cut-off value used to assign a gene superfamily was set as $53.3 \%^{49}$.

Reduction alkylation and trypsin digestion. Aliquots of collected venom $(50 \mu \mathrm{g})$ were lyophilized and reconstituted in $50 \mu \mathrm{L}$ of freshly prepared $100 \mathrm{mM} \mathrm{NH}_{4} \mathrm{HCO}_{3}$ in $30 \%$ acetonitrile at $\mathrm{pH} 8$ prior to reduction and alkylation using the previously described triethylphosphine/iodoethanol protocol ${ }^{50}$. Sigma proteomics sequencing grade trypsin was used for enzyme digestion of reduced and alkylated peptides as described ${ }^{27,29}$.

Mass spectrometry (MS). LC-ESI-MS/MS. Native injected and dissected venoms, reduced alkylated venoms and trypsin digested venoms were centrifuged $(12,000 \times g)$ to remove particulate matter prior to liquid chromatography-electrospray mass spectrometry (LC-ESI-MS) performed on an Sciex TripleTOF 5600 instrument coupled to a Shimadzu 30 series HPLC. HPLC separation was achieved on a Zorbax C18 $4.6 \times 150 \mathrm{~mm}$ column using a linear $1.3 \% \mathrm{~B}$ (acetonitrile/ $0.1 \%$ formic acid (aq) $\mathrm{min}^{-1}$ gradient at a flow rate of $0.2 \mathrm{ml} \mathrm{min}^{-1}$ over $90 \mathrm{~min}$. The gradient is optimised to elute the hydrophilic components in the beginning and hydrophobic protein like components towards the end of the run to capture the toxin peptides eluting in between. A cycle of one full scan of the mass range (MS) $(300-2000 \mathrm{~m} / z)$ followed by multiple tandem mass spectra (MS/MS) was applied using a rolling collision energy relative to the $\mathrm{m} / z$ and charge state of the precursor ion up to a maximum of $80 \mathrm{eV}$.

ConoServer and ProteinPilot search of LC/MS and LC/MS/MS data. LC-ESI-MS reconstruction of the native venom samples were performed using Analyst LCMS reconstruct BioTools (Framingham, MA, USA) with the mass range set to $800-10,000 \mathrm{Da}$, a mass tolerance of $0.2 \mathrm{Da}$, and $\mathrm{S} / \mathrm{N}$ threshold set to 10 . The LC/MS reconstruct compute the monoisotopic masses and the list of monoisotopic masses between 800 and 10,000 Da were compared between the venom duct sections and specimens. The monoisotopic mass lists were submitted to "remove duplicates" tool of ConoServer to remove duplicated masses ( 0.5 Da mass tolerance $)^{48}$. To increases the chances of detecting post translationally modified conotoxins and to complement the ProteinPilot search "differential PTM mass" tool of ConoServer was used to calculate the monoisotopic masses of the identified mature conotoxins of the transcriptome with predicted PTMs. Then these calculated monoisotopic masses with predicted PTMs were matched to the duplicate removed monoisotopic mass list obtained from the LC-MS reconstruct. The precision level was set to $0.25 \mathrm{Da}$ for automatic matching search.

The ProteinPilot 5.0 software (SCIEX, Framingham, MA, USA) was used to search the LC-ESI-MS/MS mass lists (mass tolerance of $0.05 \mathrm{Da}$ ) to identify precursor ions in reduced/alkylated and reduced/alkylated and reduced/alkylated-trypsin digested venom extracts from three venom duct sections of all three specimens. These mass data bases from each segment were separately matched against the $C$. striatus venom duct transcriptome sequences (370) obtained for all venom duct sections. The ProteinPilot Search was done separately using the reduced/alkylated and reduced/alkylated-trypsin digested venom extracts. Posttranslational modifications (PTM) used in the search covered amidation, deamidation, hydroxylation of proline and valine, oxidation of methionine, carboxylation of glutamic acid, cyclization of $\mathrm{N}$-terminal glutamine (pyroglutamate), bromination of tryptophan, sulfation of tyrosine. Additionally O-glycosylation PTMs was included in the search as typical sugar ions associated with glycosylation was identified in the MS spectrum (supplementary Figure S4). The threshold confidence value for accepting identified spectra was set to 99 and identified fragment masses were searched manually to confirm assignment. Given the challenges sequencing venom peptides with PTMs from proteomic data, we have relied on venom transcriptomic analysis to determine the distribution of venom peptides across the venom duct, with support from complementary proteomic identification of major toxins and toxin-related masses.

MALDI spot imaging. The extracts of the duct sections were analysed using an Ultraflex III TOFTOF (time-of-flight) mass spectrometer (Bruker Daltonics, Bremen, Germany) equipped with a $200 \mathrm{~Hz}$ all-solidstate laser (SmartBeam) and controlled by the FlexControl 2.4 software package using a previously reported method for cone snail venom analysis ${ }^{4}$. To analyse peptides with a mass range of $1000 \mathrm{Da}$ and 10,000 Da, Ultraflex III was operated in both linear-positive and reflectron-positive mode using CHCA as a matrix. Spectra calibration was performed externally using a peptide calibration mixture (206195, Bruker Daltonics, Bremen, Germany). A CHCA solution was made by the dilution of acetone saturated with CHCA 1 in 10 with an acetone:acetonitrile:water (6:3:1) solution. The raw samples were diluted 1 in 100 with $0.1 \%$ TFA, and $2 \mathrm{ml}$ of diluted matrix solution mixed with $1 \mathrm{ml}$ sample and spotted onto a polished steel target. For all samples, 400 shots were acquired using a random walk function at a laser frequency of $200 \mathrm{~Hz}$ and saved, with 10 replicates of each sample averaged ${ }^{4}$. Data were loaded into Clinprot Tools (Bruker Daltonics, Bremen, Germany) to visualize the 8 individual duct sections and injected predatory and defensive venoms in 'gel view' using a colorimetric gradient to show the abundance of the components in respective fractions.

Mass spectrometric data visualisation. Reconstructed mass lists from LC-ESI-MS runs of the native injected and dissected venom samples were further processed to remove $\mathrm{Na}^{+}$and $\mathrm{K}^{+}$adducts and remove duplicate masses using the embedded tools in ConoServer ${ }^{45}$. The processed LC/MS mass lists containing the monoisotopic mass, retention time and relative intensity were imported into the MarkerView (version 1.3.1) software 
(Sciex, Framingham, MA) to generate the proteome matrix comprising a list highly expressing peptide masses present at least in two samples across the venom duct extracts (distal, central and proxomal) of three specimens ( $\mathrm{A}, \mathrm{B}$ and $\mathrm{C}$ ). Relative intensities (percentage of maximum) were generated as a percentage of the most abundant peptide in each individual venom using Analyst (version 1.6) software, with unique masses aligned according to the retention time and filtered to remove background ions. Data alignment algorithms in MarkerView software were then applied to compensate for minor variations in mass and retention time to ensure the same compounds were accurately identified across the samples using a noise of threshold 10, minimum spectral peak width of $5 \mathrm{ppm}$, maximum RT peak width at 100 scans, a retention time tolerance of $0.5 \mathrm{~min}$, a mass tolerance of $25 \mathrm{ppm}$, and a maximum number of peaks to $1000^{25}$. The generated peak list and their relative abundances was used as the data matrix (supplementary Table S9) for principal component analysis (PCA) to visualize the clustering patterns of venom duct sections in replicated specimens in a Scores plot.

\section{Data avaialbility}

Data files of the reduced alkylated and reduced alkylated-trypsin digested samples are deposited in the Proteomics Identification Database (PRIDE) of EMBL-EBI (accession number PXD026194). Raw data files of the specimens A venom duct transcriptomic sequences are deposited in Sequence Read Archive (SRA) of NCBI (accession number PRJNA730990).

Received: 6 October 2020; Accepted: 27 May 2021

Published online: 24 June 2021

\section{References}

1. MolluscaBase (2020). MolluscaBase. Conus Linnaeus, 1758. Accessed through: World Register of Marine Species at: http://www. marinespecies.org/aphia.php? $\mathrm{p}=$ taxdetails\&id=137813. Accessed 19 Nov 2020.

2. Lewis, R. J., Dutertre, S., Vetter, I. \& Christie, M. J. Conus venom peptide pharmacology. Pharm. Rev. 64(2), 259-298 (2012).

3. Jin, A. H. et al. Conotoxins: Chemistry and biology. Chem. Rev. 119(21), 11510-11549 (2019).

4. Dutertre, S. et al. Evolution of separate predation-and defence-evoked venoms in carnivorous cone snails. Nat. Commun. 5(1), $1-9$ (2014).

5. Jin, A. H. et al. Transcriptome and proteome of Conus planorbis identify the nicotinic receptors as primary target for the defensive venom. Proteomics 15(23-24), 4030-4040 (2015).

6. Jin, A. H. et al. $\delta$-Conotoxin SuVIA suggests an evolutionary link between ancestral predator defence and the origin of fish-hunting behaviour in carnivorous cone snails. Proc. R. Soc. 282(1811), 20150817 (2015).

7. Prashanth, J. R., Dutertre, S. \& Lewis, R. J. Pharmacology of predatory and defensive venom peptides in cone snails. Mol. Biosyst. 13(12), 2453-2465 (2017).

8. Prashanth, J. R. et al. The role of defensive ecological interactions in the evolution of conotoxins. Mol. Ecol. 25(2), 598-615 (2016).

9. Abalde, S., Tenorio, M. J., Afonso, C. M. \& Zardoya, R. Conotoxin diversity in Chelyconus ermineus (Born, 1778) and the convergent origin of piscivory in the Atlantic and Indo-Pacific cones. Genome Biol. Evol. 10(10), 2643-2662 (2018).

10. Olivera, B. M., Seger, J., Horvath, M. P. \& Fedosov, A. E. Prey-capture strategies of fish-hunting cone snails: behavior, neurobiology and evolution. Brain Behav. Evol. 86(1), 58-74 (2015).

11. Monnier, E., Limpalaër, L., Robin, A. \& Roux, C. A. Vol. 1 and 2 of Taxonomic Iconography of Living Conidae (ConchBooks, 2018).

12. Puillandre, N. et al. Molecular phylogeny and evolution of the cone snails (Gastropoda, Conoidea). Mol. Phylogenet. Evol. 78, 290-303 (2014).

13. Puillandre, N., Duda, T. F., Meyer, C., Olivera, B. M. \& Bouchet, P. One, four or 100 genera? A new classification of the cone snails. J. Molluscan Stud. 81(1), 1-23 (2015).

14. Pi, C. et al. Analysis of expressed sequence tags from the venom ducts of Conus striatus: focusing on the expression profile of conotoxins. Biochimie 88(2), 131-140 (2006).

15. Terrat, Y. et al. High-resolution picture of a venom gland transcriptome: Case study with the marine snail Conus consors. Toxicon 59(1), 34-46 (2012).

16. Himaya, S. W. A. et al. Comparative venomics reveals the complex prey capture strategy of the piscivorous cone snail Conus catus. J. Proteome Res. 14(10), 4372-4381 (2015).

17. Pardos-Blas, J. R., Irisarri, I., Abalde, S., Tenorio, M. J. \& Zardoya, R. Conotoxin diversity in the venom gland transcriptome of the magician's cone, Pionoconus magus. Mar. Drugs 17(10), 553 (2019).

18. Pope, J. E. \& Deer, T. R. Ziconotide: A clinical update and pharmacologic review. Expert Opin. Pharmacother. 14(7), 957-966 (2013).

19. Lewis, R.J. Conotoxins: Molecular and therapeutic targets. in Marine Toxins as Research Tools 45-65 (Springer, 2009)

20. Berecki, G. et al. Analgesic $\omega$-conotoxins CVIE and CVIF selectively and voltage-dependently block recombinant and native N-type calcium channels. Mol. Pharmacol. 77(2), 139-148 (2010).

21. Casewell, N. R., Wüster, W., Vonk, F. J., Harrison, R. A. \& Fry, B. G. Complex cocktails: The evolutionary novelty of venoms. Trends Ecol. Evol. 28(4), 219-229 (2013).

22. Schendel, V., Rash, L. D., Jenner, R. A. \& Undheim, E. A. The diversity of venom: the importance of behavior and venom system morphology in understanding its ecology and evolution. Toxins 11(11), 666 (2019).

23. Davis, J., Jones, A. \& Lewis, R. J. Remarkable inter-and intra-species complexity of conotoxins revealed by LC/MS. Peptides 30(7), 1222-1227 (2009).

24. Rodriguez, A. M., Dutertre, S., Lewis, R. J. \& Marí, F. Intraspecific variations in Conus purpurascens injected venom using LC/ MALDI-TOF-MS and LC-ESI-TripleTOF-MS. Anal. Bioanal. Chem. 407(20), 6105-6116 (2015).

25. Himaya, S. W. A., Marí, F. \& Lewis, R. J. Accelerated proteomic visualization of individual predatory venoms of Conus purpurascens reveals separately evolved predation-evoked venom cabals. Sci. Rep. 8(1), 1-8 (2018).

26. Dutt, M. et al. Venomics reveals venom complexity of the piscivorous cone snail, Conus tulipa. Mar. Drugs 17(1), 71 (2019).

27. Jin, A. H. et al. Transcriptomic messiness in the venom duct of Conus miles contributes to conotoxin diversity. Mol. Cell. Prot. 12(12), 3824-3833 (2013).

28. Jakubowski, J. A., Kelley, W. P., Sweedler, J. V., Gilly, W. F. \& Schulz, J. R. Intraspecific variation of venom injected by fish-hunting Conus snails. J. Exp. Biol. 208(15), 2873-2883 (2005).

29. Dutertre, S. et al. Deep venomics reveals the mechanism for expanded peptide diversity in cone snail venom. Mol. Cell. Prot. 12(2), 312-329 (2013).

30. Hocking, H. G. et al. Structure of the O-glycosylated conopeptide CcTx from Conus consors venom. Chem-Eur. J. 19(3), 870-879 (2013). 
31. Aman, J. W. et al. Insights into the origins of fish hunting in venomous cone snails from studies of Conus tessulatus. Proc. Natl. Acad. Sci. U.S.A. 112(16), 5087-5092 (2015).

32. Neves, J. L. et al. Characterization of the first conotoxin from Conus ateralbus, a vermivorous cone snail from the Cabo Verde archipelago. Mar. Drugs 17(8), 432 (2019).

33. Jin, A. H. et al. $\delta$-Conotoxin SuVIA suggests an evolutionary link between ancestral predator defence and the origin of fish-hunting behaviour in carnivorous cone snails. Proc. R. Soc. B Biol Sci. 282(1811), 20150817 (2015).

34. Biass, D. et al. Comparative proteomic study of the venom of the piscivorous cone snail Conus consors. J. Prot. 72(2), 210-218 (2009).

35. Kelley, W. P., Schulz, J. R., Jakubowski, J. A., Gilly, W. F. \& Sweedler, J. V. Two toxins from Conus striatus that individually induce tetanic paralysis. Biochemistry 45(47), 14212-14222 (2006).

36. Himaya, S. W. A. et al. Venomic interrogation reveals the complexity of Conus striolatus venom. Aust. J. Chem. 73 (4), 357 (2020).

37. Puillandre, N., Watkins, M. \& Olivera, B. M. Evolution of Conus peptide genes: duplication and positive selection in the A-superfamily. J. Mol. Evol. 70(2), 190-202 (2010).

38. Lluisma, A. O., Milash, B. A., Moore, B., Olivera, B. M. \& Bandyopadhyay, P. K. Novel venom peptides from the cone snail Conus pulicarius discovered through next-generation sequencing of its venom duct transcriptome. Mar. Genomics 5, 43-51 (2012).

39. Teichert, R. W., Jacobsen, R., Terlau, H., Yoshikami, D. \& Olivera, B. M. Discovery and characterization of the short $\kappa$ A-conotoxins: A novel subfamily of excitatory conotoxins. Toxicon 49(3), 318-328 (2007).

40. Santos, A. D., McIntosh, J. M., Hillyard, D. R., Cruz, L. J. \& Olivera, B. M. The A-superfamily of conotoxins structural and functional divergence. J. Biol. Chem. 279(17), 17596-17606 (2004).

41. Hopkins, C. et al. A new family of Conus peptides targeted to the nicotinic acetylcholine receptor. J. Biol. Chem. 270(38), 2236122367 (1995).

42. Teichert, R. W. et al. Definition and characterization of the short $\alpha$ A-conotoxins: A single residue determines dissociation kinetics from the fetal muscle nicotinic acetylcholine receptor. Biochemistry 45(4), 1304-1312 (2006).

43. Teichert, R. W. et al. aA-Conotoxin OIVA defines a new aA-conotoxin subfamily of nicotinic acetylcholine receptor inhibitors. Toxicon 44(2), 207-214 (2004).

44. Duda, T. F. \& Palumbi, S. R. Molecular genetics of ecological diversification: Duplication and rapid evolution of toxin genes of the venomous gastropod Conus. Proc. Natl. Acad. Sci. USA 96(12), 6820-6823 (1999).

45. Holding, M. L., Biardi, J. E. \& Gibbs, H. L. Coevolution of venom function and venom resistance in a rattlesnake predator and its squirrel prey. Proc. R. Soc. B: Biol. Sci. 283(1829), 20152841 (2016).

46. Sunagar, K. \& Moran, Y. The rise and fall of an evolutionary innovation: Contrasting strategies of venom evolution in ancient and young animals. PLoS Genet. 11(10) (2015).

47. Prashanth, J. R. \& Lewis, R. J. An efficient transcriptome analysis pipeline to accelerate venom peptide discovery and characterisation. Toxicon 107, 282-289 (2015).

48. Kaas, Q., Yu, R., Jin, A. H., Dutertre, S. \& Craik, D. J. ConoServer: Updated content, knowledge, and discovery tools in the conopeptide database. Nucleic Acids Res. 40(D1), D325-D330 (2012).

49. Lavergne, V. et al. Systematic interrogation of the Conus marmoreus venom duct transcriptome with ConoSorter reveals 158 novel conotoxins and 13 new gene superfamilies. BMC Genomics 14(1), 708 (2013).

50. Hale, J. E., Butler, J. P., Gelfanova, V., You, J. S. \& Knierman, M. D. A simplified procedure for the reduction and alkylation of cysteine residues in proteins prior to proteolytic digestion and mass spectral analysis. Anal. Biochem. 333(1), 174-181 (2004).

\section{Acknowledgements}

This work was supported by ARC Project Grants (to RJL and PFA), UQ postdoctoral fellowships from The University of Queensland (SWAH) and NHMRC Research Fellowships (to RJL and PFA). The AB SCIEX 5600 mass spectrometer was supported by ARC LIEF. We thank Alun Jones from the IMB mass spectrometry facility, Gregory Bailey from IMB Sequencing facility and Jo Mulders from SCIEX Queensland for their technical help. We also thank Sebestien Dutertre for his valuable inputs in the early stages of this project and Dr. Lotten Ragnarsson for help with RNA extraction.

\section{Author contributions}

S.W.A.H. Study conceptualisation, experimental design, transcriptomic and proteomic data analysis, manuscript writing; A.H.J. RNA analysis, manuscript editing; B.H. MALDI mass spectrometry imaging, manuscript editing; S.K.R. Transcriptomic data assembly and analysis, manuscript editing; P.F.A. manuscript editing and funding; R.J.L. Study conceptualisation, data visualisation, manuscript writing and funding.

\section{Competing interests}

The authors declare no competing interests.

\section{Additional information}

Supplementary Information The online version contains supplementary material available at https://doi.org/ 10.1038/s41598-021-91919-4.

Correspondence and requests for materials should be addressed to R.L.

Reprints and permissions information is available at www.nature.com/reprints.

Publisher's note Springer Nature remains neutral with regard to jurisdictional claims in published maps and institutional affiliations. 
(c) (i) Open Access This article is licensed under a Creative Commons Attribution 4.0 International cc) License, which permits use, sharing, adaptation, distribution and reproduction in any medium or format, as long as you give appropriate credit to the original author(s) and the source, provide a link to the Creative Commons licence, and indicate if changes were made. The images or other third party material in this article are included in the article's Creative Commons licence, unless indicated otherwise in a credit line to the material. If material is not included in the article's Creative Commons licence and your intended use is not permitted by statutory regulation or exceeds the permitted use, you will need to obtain permission directly from the copyright holder. To view a copy of this licence, visit http://creativecommons.org/licenses/by/4.0/.

(C) Crown 2021 